\title{
Obstacles à la fondation d'une relation de coéducation entre parents et enseignants
}

\section{Annie Lasne}

Les dispositifs de coéducation qui concourent à renforcer la coopération entre les parents et l'école sous-entendent accord entre protagonistes, égalité des partenaires et prise en compte des compétences de chacun. L'article proposé prétend contribuer à éclairer les difficultés liées à l'étape de fondation d'une relation coéducative. Le dispositif étudié réunit des parents d'origine populaire et les acteurs scolaires d'un collège en France. Il est médiatisé par un intervenant social. L'analyse discute les écarts et décalages entre conviction en la collaboration et capacité à dépasser ses présupposés envers l'autre, écoute bienveillante et prise en considération de la valeur du partenaire, dévoilement personnel et investissement de son rôle de coéducateur.

\section{Introduction}

La loi française d'orientation sur l'éducation du 10 juillet 1989 a consacré la notion de "communauté éducative» (Ministère de l'Éducation nationale, de l'Enseignement supérieur et de la Recherche, 2016). Cette communauté réunit, selon le principe déclaré de coopération entre égaux, les parents d'élèves, les acteurs de l'éducation de l'établissement scolaire mais également ceux qui, hors de l'établissement, participent à l'accomplissement de ses missions. Depuis cette époque, le partenariat entre école et familles est prôné, mais dans les faits, le rôle institué des parents reste le plus souvent celui d'auxiliaires de l'école, de parents auxquels l'institution demande d'agir sous le contrôle des enseignants (Périer, 2005). C'est ainsi que la moitié seulement des projets éducatifs incluant les familles dans l'enseignement primaire est réellement co-construite avec les parents (Fotinos, 2014).

Ces dernières années, la réflexion sur les conditions d'une collaboration constructive a progressé en direction de dispositifs résolument coéducatifs. La loi du 8 juillet 2013 (Ministère de l'Éducation nationale, de l'Enseignement supérieur et de la Recherche, 2013a), d'orientation et de programmation pour la refondation de l'école de la République, fait de la relation entre l'école et les 
parents l'une des clés de la lutte contre l'échec et les inégalités scolaires d'origine sociale. On trouve ainsi précisé dans l'annexe à cette loi: «La promotion de la coéducation est un des principaux leviers de la refondation de l'école. Elle doit trouver une expression claire dans le système éducatif et se concrétiser par une participation accrue des parents à l'action éducative [...].» (p. 25). Pour la première fois, le cas des parents les moins familiers de l'école est évoqué: «Il s'agit aussi d'accorder une attention particulière aux parents les plus éloignés de l'institution scolaire, par des dispositifs innovants et adaptés» (p. 25).

En cohérence avec ces recommandations, des dispositifs tels que le «café des parents» (2014) ou la «mallette des parents» (2010) ont été récemment mis en place au sein de l'institution scolaire. L'évaluation de leurs effets semble conduire à des résultats encourageants. Dans les classes où le dispositif «mallette des parents» est appliqué, un surcroît sensible d'implication institutionnelle et d'implication à la maison de la part des familles, moins de convocations de l'administration et un meilleur comportement des élèves sont constatés (Gurgand, 2011).

La demande institutionnelle de travail avec les familles s'affirme également dans le cadre du Plan national de lutte contre le décrochage scolaire, lancé en 2014. Il s'agit de faire œuvre d' «alliance éducative», par la mise en commun des compétences et savoir-faire des professionnels et des parents, au service du jeune. Selon Pain (2012), la collaboration avec la famille constitue même la ligne centrale de la prévention. Le premier bilan du Plan, dressé au $1{ }^{\mathrm{er}}$ décembre 2015, montre que le nombre de décrocheurs et de jeunes de 18 à 24 ans sans diplôme a baissé. La preuve, d'après la ministre de l'Éducation nationale, qu'il convient de poursuivre le déploiement de la collaboration pour vaincre collectivement ce fléau (Vallaud-Belkacem, 2015).

Afin de renouer spécifiquement avec les parents les plus à distance de l'école, Valérie Corre, dans son rapport sur les relations entre l'école et les parents présenté en 2014 à l'Assemblée nationale, engage l'institution scolaire à aller encore plus loin. La rapporteure recommande à l'école de prendre appui sur les dispositifs de soutien à la parentalité existants, tels les Réseaux d'écoute, d'appui et d'accompagnement des parents [REAAP] ou encore les Programmes de réussite éducative [PRE]. Dans le même sens, la circulaire interministérielle du 15 octobre 2013 (Ministère de l'Éducation nationale, de l'Enseignement supérieur et de la Recherche, 2013b) souligne que le développement des partenariats, en particulier avec les associations et par exemple à l'occasion de travaux de "groupes de pairs», est de nature à favoriser le croisement des regards et des savoirs, des professionnels et des parents.

De fait, le recours à la participation d'un tiers, «facilitateur» de la relation école-famille, est de plus en plus souvent envisagé. Son rôle apparaît nécessaire à l'accompagnement des parents dans le développement de leur "pouvoir d'agir» éducatif (Le Bossé, 2003) et à celui des enseignants dans l'attitude de «reconnaissance» des droits, des attentes et des savoirs des familles (Pair, 1998). En réponse aux dérives constatées à propos du partenariat, qui parfois conduit à 
l'évitement de la discussion, il est attendu que le tiers aide à la prise de conscience des représentations de chacun et à leur dépassement. Sur le terrain, des actions contribuant à soutenir la fonction parentale sans stigmatisation, se développent. Plusieurs projets d'envergure, par exemple portés par l'association Agir tous pour la dignité-quart monde [ATD-quart monde] et par les Universités populaires de parents [UPP], en témoignent. Les travaux conduits génèrent des propositions d'actions de coéducation concrètes.

\section{Des principes coéducatifs connus, mais des conditions de réalisation à expliciter}

Selon De Singly (2010), la coéducation entre école et familles suppose l'existence d'une «société éducative démocratique» au sein de laquelle la responsabilité de l'éducation de l'enfant est partagée entre acteurs. Avec les mêmes droits et devoirs pour chacun, seule une telle "société» permet la mise en ouvre d'une démocratie participative et garantit aux parents la possibilité de parler et non seulement d'écouter.

L'accord entre parents et enseignants sur la définition de l'acte éducatif constitue une seconde condition. Si éduquer signifie répondre aux besoins de développement de l'enfant, ces besoins étant de natures diverses, cognitive, affective, sociale, idéologique (Humbeeck, Lahaye, Balsamo \& Pourtois, 2006), ni les parents ni les enseignants ne peuvent parvenir seuls à les satisfaire. Coéduquer devient incontournable. L'expérience et le savoir des parents, bien que profanes, contribuent au même titre que le savoir des professionnels à l'acte d'éduquer. Le "paradigme de la coéducation" proposée par les chercheurs de l'université de Mons-Hainaut en Belgique résume ce second principe: «L'enseignant et le parent coéduquent lorsque l'un et l'autre prennent en considération les besoins fondamentaux du développement psycho-social de l'enfant tout en préservant les savoirs respectifs [...] et les champs d'enseignement [...] de chacun» (Humbeeck et al., p. 653).

Enfin, la relation coéducative implique que l'enfant et son cheminement soient la principale source de l'échange entre partenaires (Humbeeck et al.). Ainsi centré sur l'enfant en tant que sujet, ce processus de communication évite que la discussion ne s'égare sur la mise en question des contenus enseignés et des méthodes de l'enseignant de la part des parents ou sur les savoirs transmis par le milieu familial de la part des enseignants.

Pour autant, connaître les principes essentiels à l'installation d'une relation coéducative ne suffit pas à garantir la réussite de l'entreprise. Quand bien même chaque acteur éducatif est convaincu de la nécessité d'une coopération renforcée, la question de ses modalités concrètes de mise en œuvre reste posée. En effet, l'accord sur des objectifs généraux, «la réussite des élèves et la formation d'adultes citoyens responsables» (Auduc, 2007), garantit-il à lui seul le succès 
de la rencontre entre éducateurs? L'usage d'un vocabulaire commun assure-t-il un accord sur le sens que recouvrent les termes de coéducation, de partage de la responsabilité éducative? Au-delà, la volonté de s'engager dans une démarche coéducative est-elle suffisante pour co-construire? Enfin, l'intérêt mutuel à coopérer suffit-il pour dépasser les attentes singulières et concilier les fonctionnements identitaires des uns et des autres?

\section{Un dispositif coéducatif sous la loupe}

Le support de l'étude présentée ici est une recherche-action en cours qui vise le rapprochement entre un groupe de professionnels d'un collège (élèves de 11 à 15 ans) en France: enseignants et personnels d'encadrement, et un groupe de parents d'élèves, d'origine populaire. Le projet est à l'initiative d'une intervenante sociale, coordonnatrice des actions collectives en direction des familles dans un centre culturel proche du collège, et à laquelle les parents qu'elle rencontre expriment régulièrement leur relation difficile avec l'établissement scolaire ainsi que leur manque de ressources quant au suivi de la scolarité des enfants. Confortée par le vif intérêt manifesté par le principal du collège à entrer en relation avec des parents que l'école connaît peu, l'intervenante sociale propose aux parents qu'elle côtoie et à l'équipe pédagogique un projet de rencontre en vue d'une acculturation réciproque. À terme, le but est d'engager ces différents acteurs dans l'élaboration d'actions éducatives conjointes au service des élèves. Il s'agit, en outre, d'établir ensemble un climat relationnel qui encourage les parents actuellement éloignés de l'établissement à s'en rapprocher. Ainsi défini, huit parents, toutes des mères, acceptent d'entrer dans le projet. Six parents appartiennent aux catégories sociales défavorisées (ouvrier, sans emploi), les deux autres aux professions intermédiaires. Sept parents sur huit sont d'origine étrangère (Maroc, Tunisie). Le principal du collège, la conseillère principale d'éducation, l'assistante sociale scolaire et cinq enseignants se portent volontaires. En début de travail, les parents et le corps professoral ne se connaissent pas ou s'identifient seulement.

La première partie du projet est constituée temporellement de deux phases. La première a consisté en la fédération des parents et des professionnels autour $\mathrm{du}$ projet. Cinq rencontres mensuelles ont eu lieu en groupes séparés, d'une part avec les parents et d'autre part avec les acteurs du collège. Elles ont d'abord favorisé l'expression de chacun sur la relation école-familles et ainsi permis d'évaluer les différences et oppositions entre représentations. Elles ont par la suite contribué à identifier les questionnements et les problèmes à résoudre et de ce fait conduit les participants à se décentrer de leur propre analyse. Enfin, elles ont engagé chaque groupe à se projeter dans les contenus d'un travail commun. La seconde phase du travail a été consacrée à l'entrée en relation. Trois rencontres communes ont été mises en œuvre, les deux premières au collège sur invitation 
des professionnels et la troisième au centre culturel et social à l'initiative des parents. Leur premier objectif était d'installer la reconnaissance mutuelle des compétences éducatives de chacun. Le second objectif visait la construction du groupe en tant que collectif capable de dépasser les différences premières pour pouvoir réfléchir ensemble à des pistes d'action. Cette première partie du projet a duré huit mois. Toutes les séances ont été animées par l'intervenante sociale dans une perspective participative. Son rôle était de proposer un cadre de travail, d'apporter des outils pour le fonctionnement du groupe et d'accompagner ce dernier à atteindre les objectifs fixés. Elle n'intervenait pas sur le contenu des échanges. Le chercheur a participé à l'ensemble du travail en tant que personne ressource ayant la possibilité d'enrichir les débats du groupe mais ne portant aucun jugement.

Le matériau analysé est constitué des observations de l'ensemble des rencontres. Elles retracent les thématiques abordées, les types de participation et leur fréquence, les modes de communication utilisés ainsi que le climat des échanges. Des entretiens individuels ont, par ailleurs, été menés avec chaque acteur impliqué dans le projet. Ils ont porté sur les thèmes suivants: la définition du rôle de l'école et de la famille dans la relation école-famille; l'appréciation portée, antérieurement au projet, sur les relations entretenues entre enseignants et parents; les représentations et attentes des enseignants à l'égard des familles et réciproquement; les ambitions des uns et des autres vis-à-vis d'un tel projet; les conditions et occasions qui pourraient faciliter l'atteinte des objectifs.

\section{Quelques éclairages sur l'étape de construction de la relation de coéducation}

Le modèle coéducatif fondé sur la réciprocité entre parents et enseignants nécessite un partage total des expériences et des connaissances (Bouchard, 1998). Il repose sur des principes d'appropriation (empowerment) et d'autodétermination (enabling). L'appropriation correspond à l'actualisation de ses propres ressources et compétences alors que l'autodétermination renvoie à l'habileté de définir son rôle et de déterminer la manière dont on entend collaborer. Les exigences d'un tel modèle laissent envisager les difficultés qui pourraient faire obstacle à son application. Dans le cas présent, la volonté partagée de participer à un programme de rencontres devrait, selon nous, favoriser les échanges entre acteurs dans un climat convivial et éviter la confrontation récurrente des approches parentale et scolaire. Une communication bidirectionnelle est attendue, donnant l'occasion aux parents et aux enseignants d'échanger sur leurs préoccupations éducatives (Favre, 2004). Cependant, en raison de la différence profonde des attentes et des valeurs entre "école» et "maison", nous pensons que les enseignants peineront à prendre en considération le parent en tant que partenaire et que les parents n'investiront que difficilement leur rôle de co-acteur du partenariat. 


\section{Les rencontres entre groupes de pairs}

\section{Croire en soi et croire en l'autre}

Les enseignants étudiés croient en l'intérêt du travail conjoint parents-enseignants, «d'égal à égal». Ils expriment tous la nécessité d' «être unis dans l'effort» et qu'il est essentiel d' «aller ensemble dans la même direction pour réussir». L'idée d'entrer en relation avec les parents ne génère donc pas d'opposition de la part des enseignants. Aucun doute n'est émis quant à la légitimité de l'entreprise. Les propos tenus rejoignent les résultats d'Asdih (2012) qui montrent que les enseignants, soutenus par les politiques éducatives et la conviction que cela concourt à la réussite scolaire de l'élève, considèrent la collaboration avec les parents primordiale.

Toutefois, pour les acteurs étudiés, il convient, certes, «d'entendre les parents», mais surtout de les faire contribuer à la résolution des difficultés d'apprentissage ou de comportement que pourrait poser l'enfant. Plusieurs s'accordent sur le fait qu'il s'agit de "préparer le terrain au cas où" et de sensibiliser les parents à leur rôle d'éducateur responsable, y compris pour ce qui se passe à l'école. En ce sens, comme l'indiquent Chartier et ses associés (Chartier, Rufin \& Pelhate, 2014), la collaboration devient un moyen de négocier des actions éducatives futures, au cas où la situation de l'enfant viendrait à le nécessiter.

Aller au contact des parents n'inquiète qu'une minorité d'enseignants. Ceux-ci expriment la crainte de rencontrer des parents qui en "veulent aux enseignants», qui ont de "mauvais rapports avec l'école» au regard de leur expérience scolaire propre. Mais pour la grande majorité des enseignants, la volonté des parents à $s$ 'inscrire dans le travail garantit leur intention constructive.

Les obstacles qui pourraient limiter les parents à entrer en relation avec les enseignants sont peu évoqués. Que la relation soit difficile à établir pour les familles en raison d'un éventuel sentiment de stigmatisation ou d'un rapport à l'école douloureux est évoqué mais les conséquences s'en trouvent minimisées du fait de la participation volontaire des familles au projet. Aux yeux des professeurs, les parents apparaissent disposés à coopérer. Périer (2005) le signale dans ses travaux, cet attribut de "parents coopératifs» est interprété comme une garantie de l'entente mutuelle.

Les parents appellent, eux-aussi, la collaboration de leurs vœux. Mais pour eux, la rencontre est une réussite en soi. Les parents présupposent que si la rencontre est effective, les problèmes qu'ils rencontrent se dissiperont nécessairement, «si on arrive à parler aux professeurs, ce sera gagné!». D'emblée, ils font référence à leur expérience réussie lorsque l'enfant était scolarisé en école maternelle. Ils en tirent qu'un lien fort existe entre le succès scolaire et le fait que l'école soit abordable. Les parents étudiés ne se considèrent donc pas éloignés de l'école, mais tenus à distance par elle. Dans son étude des attentes des familles à l'égard de l'école, Prévôt (2008) établit le même constat: considérée inaccessible par les parents, l'école devient responsable des incompréhensions, des différends avec 
les enseignants. Plus que la nature du message échangé, c'est donc l'existence de l'échange qui prévaut. Le formalisme des relations que le collège propose vient en contradiction avec les attentes parentales.

Au contraire des enseignants, les parents formulent des doutes quant à la possibilité d'établir un échange. Ils s'en sentent responsables. Ils indiquent avoir peur de rencontrer les professeurs, avoir «la boule au ventre» pour certains, de franchir les portes du collège. Ils expriment la crainte d' «être perdus», tant dans l'espace que dans les propos qu'on leur tiendra. Ils disent leur appréhension de ne pas savoir répondre, de «ne pas avoir les bons mots». Leur doute est en outre alimenté par l'inquiétude de devoir faire face à un collectif soudé. La crainte n'est cependant pas de ne pas avoir de place pour s'exprimer, mais plutôt de ne pas savoir s'ils vont pouvoir occuper la place qu'ils estiment leur être donnée. Les parents semblent dans l'incompréhension du rôle qu'on leur demande d'assurer. À l'instar de ce que montre Deslandes (2001), leur sentiment d'incompétence augmente en même temps que leur croyance au fait qu'ils ont à participer diminue. Les parents ont, au final, du mal à s'approprier l'idée que leurs avis sont souhaités et qu'ils sont partenaires à part entière de l'école.

\section{La collaboration: opportunité ou menace}

Globalement peu inquiets à propos de l'étape d'entrée en relation avec les parents, les acteurs scolaires expriment de fortes interrogations quant au contenu et à l'organisation des rencontres. La relation est en effet envisagée comme «un long chemin», une «voie incertaine». Les enseignants craignent de ne pas «réussir» et demandent de l'aide: «comment saura-t-on que l'on est sur le bon chemin?», «qui pourra nous aider, si nous n'aboutissons pas?». Le doute sur leur capacité à faire face s'insinue d'autant plus fortement qu'ils restent majoritairement persuadés qu'ils ont le devoir «d'être à la hauteur». Chartier et ses collaborateurs formulent une remarque qui s'apparente à notre constat: «La participation des parents à ces échanges, bien qu'attendue, est perçue comme une source d'incertitude pour les enseignants qui souhaitent en maîtriser le déroulement» (Chartier, Rufin \& Pelhate, 2014, p. 44.). Habitués à des modalités d'action qui s'adossent à une expertise institutionnellement reconnue, les enseignants se trouvent dans le cas présent déstabilisés lorsqu'il s'agit de co-construire des solutions novatrices avec les familles.

En réponse au malaise ressenti, la tendance est d'abord à la protection. Les enseignants défendent l'idée que la relation avec les parents ne sera profitable que si chacun "garde sa liberté» et «fait des choix en toute sérénité». La tendance à la relativisation de la portée de l'action apparaît ensuite. Ils rappellent la nécessité de «disposer de temps» pour co-construire, qu'ils «ne sont que de passage» et encore que la pérennité du résultat est incertaine: «qu'en restera-t-il?». Enfin, pointe la tendance à la responsabilisation des parents: "les parents sont la clés, «les parents doivent nous aider». Ces réticences s'appuient sur la perception que les relations avec les familles ne sont pas le cœur du métier. Les enseignants 
pointent d'ailleurs la préparation au métier, plus centrée sur les disciplines que sur les finalités éducatives et citoyennes de l'école. Enfin, la crainte de l'échec, de ne pas réussir à satisfaire les attentes institutionnelles, montre à quel point l'exigence de rendement est associée à cette démarche de rapprochement avec les familles. Ce faisant, ce souci d'efficacité entre en opposition avec le principe de développement de la relation.

Là où les enseignants s'inquiètent de leur maîtrise relative des contenus de l'échange, les parents s'avèrent assurés dans l'énoncé de leurs questionnements. Leurs préoccupations concernent essentiellement l'enfant: son travail scolaire, son comportement et son orientation. Les interrogations formulées renvoient à des attendus concrets "comment le mettre au travail?", "comment choisir son orientation?». Cependant, par la complexité des réponses qu'elles attendent, ces questions témoignent du dénuement des parents en matière scolaire. Les parents n'adoptent donc pas une position attentiste. Leur application à établir une liste exhaustive des points à éclairer montre qu'ils envisagent la rencontre avec les enseignants comme une stratégie de recherche de réponses. Chartier et al. (2014) notent également cette attente dans leurs analyses d'entretiens individuels entre parents et enseignant.

Les familles entendues n'ont aucun doute sur l'issue positive de la coopération. Mais, c'est l'enseignant qui est perçu l'artisan de cette réussite. Il a le statut d'expert des questions scolaires: «les professeurs sauront nous répondre». Par le fait, les parents n'envisagent pas spontanément d'élaborer des solutions avec les enseignants, mais plutôt de profiter de l'expertise qu'ils leur conferent. $\mathrm{Ni}$ le contenu, ni le déroulement de l'échange ne génèrent d'interrogations de la part des parents étudiés. Il semble entendu que la rencontre leur offre un espace d'expression qu'ils doivent saisir, non pour revendiquer un statut de partenaire, mais pour bénéficier des compétences des enseignants.

\section{Les rencontres communes}

\section{Entente de surface et mésentente en profondeur}

La volonté partagée de se rencontrer contribue à la création d'un climat de confiance entre les parties. Les familles s'autorisent à s'exposer et les enseignants écoutent avec une réelle bienveillance les difficultés que les parents relatent à propos de leur quotidien de vie. Ils entendent la réalité des familles, mais semblent toutefois traiter les informations recueillies en mobilisant les deux registres de catégorisation des parents évoqués par Giuliani et Payet (2014). Les parents sont considérés, d'une part victimes des réalités sociales et d'autre part déficitaires dans leurs capacités à répondre complètement aux besoins de développement de l'enfant. Ces perceptions ont au moins deux conséquences. D'abord, elles renforcent la représentation du parent dépendant de l'école et donc légitimement disposé à coopérer. Ensuite, elles empêchent les enseignants de considérer pleinement le parent comme un partenaire éducatif. 
Quant aux parents, ils interprètent l'empathie que leur témoignent les enseignants, à la fois comme l'approbation de leurs valeurs éducatives et comme la confirmation que des solutions adaptées vont leur être proposées. Lorsque les parents livrent par exemple leurs inquiétudes quant à l'irrespect de l'autorité qu'ils pensent percevoir chez les adolescents, ils trouvent l'assentiment des enseignants qui abondent dans leur sens en faisant référence au registre de langue inapproprié que les jeunes utilisent parfois en classe. Mais lorsqu'ils affirment que des règles strictes doivent être appliquées sans concession, que la sanction caractérise une «bonne éducation», le silence en guise de réponse enseignante n'est pas interprété comme le signe d'un décalage de point de vue, mais comme la continuité de l'approbation. Si l'entente existe sur le diagnostic posé, les moyens que les enseignants mettraient en ouvre pour traiter le problème ne sont pas souvent explicités et sont peu audibles par les parents lorsqu'ils le sont. Les échanges conviviaux confortent les parents dans leur impression que les enseignants partagent leur avis et les enseignants dans leur catégorisation des parents. À l'instar de ce qu'affirment Tazouti, Flieller et Vrignaud (2005) dans leur comparaison de l'éducation parentale entre deux milieux sociaux contrastés, derrière l'accord de surface sur les objectifs éducatifs abordés, apparaissent de vraies divergences de valeurs et corrélativement de pratiques pour les atteindre.

Aux questions auxquelles les parents cherchent des réponses - le choix de l'orientation, la concentration de l'élève ou encore l'aide aux devoirs - il est attendu que les enseignants dévoilent les recettes du bon accompagnement scolaire, de ce qui va "permettre le déclic» chez l'enfant. Les parents prévoient ainsi d'obtenir des informations d'ordre "technique», d'accéder à la «boîte à outils» de l'enseignant. Ils veulent voir en lui le spécialiste en capacité de produire des conseils efficaces dont ils n'ont pas pu bénéficier jusque-là. Cette perception élémentaire du rapport au savoir se trouve, en outre, encouragée par les enseignants eux-mêmes lorsque ceux-ci recommandent de ne suivre que quelques préceptes simples, comme "prendre connaissance du carnet de correspondance» et «'sassurer que les devoirs sont faits». La convivialité de l'échange, l'entente préalable sur le besoin de coopérer ne conduit pas naturellement à l'explicitation des repères éducatifs de référence. Or, si chercher un consensus n'est sans doute pas une voie porteuse, comprendre la logique de l'autre pourrait permettre d'identifier les leviers sur lesquels chacun serait en mesure d'agir. Au lieu de cela, l'un continue de voir dans l'attitude de l'autre la confirmation de ce qu'il s'attendait à voir.

\section{Condition à l'action de coéducation ou action de coéducation}

Du point de vue des enseignants, la coopération avec les familles doit rapidement aboutir à la mise en place de dispositifs au service de tous les élèves. Ainsi, lors de la troisième rencontre, les enseignants prévoient d'organiser des réunions pour informer leurs collègues des contenus échangés ou encore envisagent de créer, pour la rentrée suivante, une classe pilote au sein de laquelle les parents seraient 
invités plus souvent qu'à l'accoutumée. Les solutions qu'ils élaborent doivent pouvoir être, à court terme, généralisables et transférables aux différentes situations rencontrées au collège. Dégager un modèle de la relation efficace entre parents et enseignants apparaît le but prioritaire, au risque de laisser les parents présents en dehors de la réflexion.

En écoutant les parents «dire» leurs difficultés et questionnements lors de la première rencontre et en soutenant cette verbalisation, les enseignants semblent avoir construit une légitimation à leur action future. La réappropriation du discours parental a permis de confirmer l'incertitude qu'ils nourrissaient quant aux compétences éducatives familiales. Et encouragés par l'établissement d'accords minimaux, les enseignants se sont sentis autorisés à faire des propositions d'actions. Dans leurs travaux, Tardif et Lessard (1999) mentionnent également que les professeurs évoquent la nécessité d'agir suite à une réinterprétation de la teneur des propos parentaux. Ainsi, la collaboration avec les familles viserait, en priorité et conformément à ce qu'établit Chauveau (2000), à établir la confiance nécessaire à l'adhésion des parents aux projets que les enseignants prévoient de se charger de construire. Cette manière de procéder est contraire aux objectifs de coopération qui demandent du temps et une prise en compte réciproque des points de vue. Au final, les parents ne paraissent pas reconnus dans leurs compétences ou, comme le souligne Payet (2008), seulement sous une forme "purement rhétorique». Si les parents ne sont pas ici tout à fait des auxiliaires au service de l'école, ils sont les bénéficiaires des actions partenariales mais en aucun cas des partenaires.

À l'inverse des enseignants, les parents étudiés ne cherchent pas à écourter l'étape d'échanges personnels au profit de l'entrée dans la conception de dispositifs. Tout au contraire, rassurés par la bienveillance des professionnels nouvellement acquise, quitter le registre individuel pourrait signifier renoncer à la satisfaction de leurs intérêts propres. Ils cherchent donc à maintenir le plus longtemps possible l'attention des enseignants, en faisant, au besoin, quelques révélations familiales. Les émotions, les sentiments guident leurs demandes et fondent les liens qu'ils souhaitent tisser avec les enseignants. Une sorte d'alliance affective est recherchée et c'est sur ce terrain qu'ils essaient d'attirer les professionnels. Giuliani et Payet (2014) montrent qu'en se laissant aller à la confidence, les parents ouvrent la frontière de la sphère privée et étendent le terrain des échanges.

Toutefois, si ce positionnement exprime de la confiance à l'égard de l'enseignant, il peut le déstabiliser, notamment si celui-ci considère que les limites de son rôle traditionnel sont dépassées. Dans la situation étudiée, face aux confidences parentales, les enseignants sont démunis. Ils en déduisent que les parents sont en demande d'aide et s'empressent de se retirer de cet espace peu sécurisant. Ils regagnent le contexte éducatif qu'ils connaissent le mieux, confortant au passage, comme le rappellent Humbeeck et ses collègues (Humbeeck et al., 2006), l'idée que rester chacun à sa place et respecter la succession des périodes 
éducatives réservées à la sphère familiale et à la sphère scolaire définit une relation de coéducation efficace.

Si les parents cherchent à faire évoluer la relation vers plus de proximité avec les enseignants, c'est aussi parce qu'ils accordent beaucoup de valeur aux échanges d'informations. La discussion, avec d'autres parents par exemple, est un recours souvent mobilisé au quotidien dans une perspective d'entreaide. En ce sens, Monceau (2014) montre que les parents éloignés de l'école considèrent que les contacts individuels sont les plus utiles des rapports. Ainsi, dans l'entreprise collaborative analysée, ces conversations semblent perçues par les parents comme une réelle action de coéducation. Du côté des enseignants, elles sont davantage considérées comme une condition à l'action de coéducation.

Enfin, les parents perçoivent que quitter l'échange individuel pour s'inscrire dans une réflexion collective risque de les mettre en difficulté. Ils redoutent de se trouver dans un registre où leurs compétences à l'argumentation pourraient manquer. Cette crainte est d'ailleurs objectivement validée lorsque les enseignants engagent seuls la réflexion à propos des dispositifs à construire. Les parents ne contestent toutefois pas cette mise à l'écart. Ils laissent même le champ libre aux enseignants, quittent la table de travail et proposent café et gâteaux. À l'issue des rencontres, les parents semblent avoir accepté de réintégrer leur rôle de subalternes. Sauf à considérer qu'ils se satisfont des premières informations reçues, ce qu'envisagent Dumoulin et ses confrères (Dumoulin, Thériault \& Duval, 2014), il reste possible, comme le suggère Périer (2007), qu'ils ne soient pas pleinement conscients que leur rôle se poursuit dans l'élaboration de projets co-construits.

\section{Conclusion}

La définition d'une relation de coéducation décrit une relation fondée sur le principe d'égale dignité entre acteurs, de complémentarité des savoirs et d'union des motivations au service de l'enfant. Le changement de regard sur l'autre et la reconnaissance de modes de fonctionnement différents du sien, en tant qu'atouts plutôt que freins à la construction d'une relation de véritable coopération, ne se décrètent cependant pas. L'expérimentation présentée dans cet écrit semble montrer que l'entrée dans la relation coéducative se heurte à plusieurs obstacles.

Les enseignants ont la conviction que la collaboration avec les parents contribue à la réussite de l'élève et ont le souci de la relation. Toutefois, ils ne semblent attribuer que peu de crédit aux capacités des parents à faire évoluer le rapport à l'apprentissage de leur enfant et ont tendance à les reléguer au rôle d'informateurs. L'échange sert en effet prioritairement à recueillir les données qui leur seront utiles pour construire les dispositifs jugés adaptés aux besoins qu'ils estiment avoir repérés. Sans doute par volonté d'être efficaces dans leurs actions, les enseignants ne cherchent pas à s'appuyer sur les compétences des parents pour co-construire. Du côté des parents, si ceux-ci sont en manque d'estime 
d'eux-mêmes, ils ont cependant des attentes concrètes et veulent rencontrer les enseignants. Pour tenter d'obtenir des réponses à leurs questions, ils recherchent les contacts individuels et utilisent le registre affectif. Mais face à la résistance des enseignants à entrer sur ce terrain, et parce qu'ils peinent à investir leur rôle de co-acteur, les parents finissent par accepter de devenir les bénéficiaires des dispositifs pensés par les enseignants.

L'expérimentation analysée nous apprend que la volonté de collaborer ne garantit pas la construction d'une relation réellement coéducative. Elle suggère que les protagonistes ont à développer leur capacité à se "relier» entre eux et au projet. Chacun a à dépasser ses présupposés, à faire un travail d'acculturation, à accepter le tâtonnement et la déstabilisation de ses repères, à investir son rôle de co-constructeur ainsi qu'à partager la responsabilité éducative. Mais amener les parents et les professionnels à changer leurs modes de relation et instaurer une nouvelle culture en la matière demande un effort soutenu et beaucoup de temps (Deslandes, 2001).

Les obstacles répertoriés nous invitent aussi à interroger le contenu des séances de coéducation mises en place. Bien que la volonté fût de réduire l'asymétrie de la relation grâce aux séances de préparation réalisées en groupes séparés, les contenus des séances communes, centrés sur la définition d'une relation de coéducation, ont donné l'avantage à l'expertise enseignante. Les parents ont alors progressivement réintégré leur statut de personnes à aider tout en confortant les enseignants dans une posture d'aidants.

Le partenariat est un idéal qui demande des modifications importantes dans les façons de penser et d'agir de chacun (Deslandes, 2001). À cette fin, il apparaît essentiel que les enseignants et personnels d'encadrement soient davantage préparés au travail avec des adultes. Dotés de compétences communicationnelles adaptées, ils seraient plus à même de dépasser leurs craintes des relations interpersonnelles. La formation gagnerait également à les aider à prendre conscience de leurs représentations et à travailler à l'élucidation des perceptions et des attentes du partenaire (Deslandes \& Bertrand, 2001). Enfin, il serait sans doute bénéfique que l'école augmente la disponibilité des enseignants pour les parents ou encore prévoie le recours à un agent de liaison qui faciliterait les interactions entre les parents et les enseignants (Deslandes, 2001). Du côté des parents, un travail spécifique visant à leur redonner un sentiment de confiance et de compétence (Périer, 2007) permettrait de les équiper et de les mobiliser pour qu'ils se constituent en sujets aptes à prendre leurs responsabilités éducatives face aux institutions (Dumoulin et al., 2014; Giuliani \& Payet, 2014). 


\section{Références et bibliographie}

Asdih, C. (2012). Collaborer avec les parents pour accompagner la scolarité? Représentations des enseignants et pratiques professionnelles. Enfances, Familles, Générations, 16, 34-52.

Auduc, J.-L. (2007). Les relations parents-enseignants à l'école primaire. Créteil: CRDP de l'académie de Créteil.

Bouchard, J.-M. (1998). Le partenariat dans une école de type communautaire. In R. Pallascio, L. Julien \& G. Gosselin (Éd.), Le partenariat en éducation. Pour mieux vivre ensemble! (pp. 19-35). Montréal: Éditions Nouvelles.

Chartier, M., Rufin, D. \& Pelhate, J. (2014). Les enseignants dans l'entretien individuel avec les parents: entre souci de la relation et exigence d'efficacité. Éducation et sociétés, 34(2), $39-54$.

Chauveau, G. (2000). Comment réussir en ZEP. Paris: Retz.

Circulaire n²013-142 du 15 octobre 2013. Renforcer la coopération entre les parents et l'école dans les territoires.

Corre, V. (2014). Rapport d'information déposé par la commission des affaires culturelles et de l'éducation sur les relations entre l'école et les parents. Paris: Assemblée nationale.

De Singly, F. (2010). Communauté éducative ou société scolaire démocratique? In P. Madiot (Éd.), Enseignants, parents, réussite des élèves: quel partenariat? (pp. 11-15). Amiens: CRDP d'Amiens et CRAP-Cahiers pédagogiques.

Deslandes, R. (2001). A vision of home-school partnership: three complementary conceptual frameworks. In F. Smit, K. van der Wolf \& P. Sleegers (Éd.), A bridge to the future. Collaboration between parents schools and communities. Nijmegen: ITS, Stichting Katholieke Universiteit te Nijmegen.

Deslandes, R. \& Bertrand, R. (2001). La création d'une véritable communauté éducative autour de l'élève: une intervention plus cohérente et des services mieux harmonisés. Rapport de recension des écrits, CQRS-MEQ action concertée. Consulté le 28 mars 2016 dans http:// www3.education.gouv.qc.ca/tragiquement/OutilsDocum/CommunEduc.pdf

Dumoulin, C., Thériault, P. \& Duval, J. (2014). Opinion parentale sur des pratiques efficaces de collaboration école-famille au primaire. La revue internationale de l'éducation familiale, 36(2), 117-140.

Favre, B. (2004). Culture commune autour des apprentissages. Résonances, (janvier), 7-9.

Fotinos, G. (2014). Face à face entre confiance et méfiance: lycée-collège et parents d'élève: une enquête quantitative auprès des directeurs d'école maternelle et élémentaire. Noisiel: Casden.

Giuliani, F. \& Payet, J.-P. (2014). Introduction. Les logiques scolaires de la proximité aux familles. Éducation et sociétés, 34(2), 5-21.

Gurgand, M. (Éd.). (2011). Quels effets attendre d'une politique d'implication des parents dans les collèges: évaluation de l'impact de la mallette des parents. Paris: Ministère de l'Éducation nationale, de la de la Jeunesse et de la Vie associative.

Humbeeck, B., Willy Lahaye, W., Balsamo, A. \& Pourtois, J.-P. (2006). Les relations école-famille: de la confrontation à la coéducation. Revue des sciences de l'éducation, 32(3), 649-664.

Le Bossé, Y. (2003). De «l'habilitation» au «pouvoir d'agir»: vers une appréhension plus circonscrite de la notion d'empowerment. Nouvelles Pratiques Sociales, 16(2), 30-51.

Ministère de l'Éducation nationale, de l'Enseignement supérieur et de la Recherche (2013). Loi $n^{\circ}$ 2013-595 du 8 juillet 2013 d'orientation et de programmation pour la refondation de l'école de la République. Consulté le 15 avril 2016 dans https://www.legifrance.gouv.fr/ affichTexte.do?cidTexte=JORFTEXT000027677984\&categorieLien=id

Ministère de l'Éducation nationale, de l'Enseignement supérieur et de la Recherche (2013b). Circulaire n²013-142 du 15 octobre 2013. Renforcer la coopération entre les parents et l'école dans les territoires. Bulletin officiel, 38. Consulté le 15 avril 2016 dans http://www. education.gouv.fr/pid25535/bulletin_officiel.html?cid_bo=74338 
Ministère de l'Éducation nationale, de l'Enseignement supérieur et de la Recherche (2016). Loi d'orientation sur l'éducation, loi n'89-486 du 10 juillet 1989. Consulté le 15 avril 2016 dans http://www.education.gouv.fr/cid101274/loi-d-orientation-sur-l-education-n-89486-du-10-juillet-1989.html

Monceau, G. (2014). Effets imprévus des dispositifs visant à rapprocher les parents éloignés de l'École. Éducation et sociétés, 34(2), 71-85.

Pain, C. (2012). Déscolariser le décrochage. In J.-L. Gilles, P. Poitvin \& C. Tièche Christinat (Éd.), Les Alliances éducatives pour lutter contre le décrochage scolaire (pp. 193-205). Berne: Peter Lang.

Pair, C. (1998). L'école devant la grande pauvreté: changer de regard sur le quart-monde. Paris: Hachette.

Payet, J.-P. (2008). Le caractère du fonctionnaire. Sur les conditions ordinaires de la reconnaissance. In J.-P. Payet \& A. Battegay (Éd.), La reconnaissance à l'épreuve: explorations socio-anthropologiques ( $p$. 103-112). Villeneuve-d'Ascq: Presses universitaires du Septentrion.

Périer, P. (2005). Ecole et familles populaires: sociologie d'un différend. Rennes: Presses Universitaires de Rennes.

Périer, P. (2007). École et familles populaires. XYZep, 26, 1-3.

Prévôt, O. (2008). Attentes des familles à l'égard de l'école: une enquête auprès de 2492 parents. In G. Pithon, C. Asdih \& S. Larivée (Éd.), Construire une "communauté éducative» (le partenariat familles-associations-écoles-universités) (pp. 37-50). Bruxelles: De Boeck université.

Tardif, M. \& Lessard, C. (1999). Le travail enseignant au quotidien. Bruxelles: De Boeck.

Tazouti, Y. Flieller, A. \& Vrignaud, P. (2005). Comparaison des relations entre l'éducation parentale et les performances scolaires dans deux milieux socioculturels contrastés (populaire et non populaire): Pratiques éducatives familiales et scolarisation. Revue française de pédagogie, 151, (1), 29-46.

Vallaud-Belkacem N. (2015). Intervention du 1er décembre 2015 dans le cadre du plan national d'actions "Tous mobilisés pour vaincre le décrochage». Consulté le 28 mars 2016 dans http:// www.education.gouv.fr/cid96061/tous-mobilises-pour-vaincre-le-decrochage-scolaire. html

Mots-clés: Coéducation, relation, enseignants, parents, obstacles 


\section{Hindernisse beim Aufbau einer koedukativen Beziehung zwischen Eltern und Lehrkräften}

\section{Zusammenfassung}

Bei koedukativen Methoden, die dazu beitragen, die Zusammenarbeit von Eltern und Schule zu verstärken, müssen sich die Protagonisten einig sein und sich gleichberechtigt begegnen. Auch sollen die Kompetenzen jedes Einzelnen berücksichtigt werden. Der vorliegende Artikel versucht, die Schwierigkeiten des Aufbaus einer koedukativen Beziehung darzustellen. Die untersuchte Methode bringt Eltern aus bescheidenen Verhältnissen mit dem Personal einer Sekundarschule in Frankreich zusammen. Ein Sozialarbeiter unterstützt das Miteinander-in-Beziehung treten. Die Studie zeigt Unterschiede und Gegensätze zwischen der Überzeugung zur Zusammenarbeit und der Fähigkeit, Vorurteile zu überwinden, wohlwollendem Zuhören und Berücksichtigung des Partners, persönlichem Erscheinen und Engagement in der Rolle eines Ko-Erziehers.

Schlagworte: Koedukation, Beziehung, Lehrer, Eltern, Hindernisse

\section{Gli ostacoli per la costruzione di una relazione di coeducazione tra genitori e figli}

Riassunto

I dispositivi di coeducazione che concorrono a rinforzare la cooperazione tra genitori e scuola implicano un accordo tra i protagonisti, l'uguaglianza dei partner e la valorizzazione delle competenze di ciascuno. Il presente articolo si propone di far luce sulle difficoltà legate al momento della costruzione della relazione coeducativa. Il dispositivo preso in considerazione riunisce genitori di origini popolari e attori di una scuola media in Francia. Tale dispositivo è mediato dall'azione di un professionista dell'intervento sociale. L'analisi proposta metterà in evidenza i divari che emergono tra la fiducia nella collaborazione e la capacità di superare i propri presupposti in favore dell'altro, l'ascolto e la presa in considerazione del valore dei partner, nonché lo svelamento personale e l'investimento del proprio ruolo di co-educatore.

Parole chiave: Coeducazione, relazioni, insegnanti, genitori, ostacoli 


\title{
Obstacles to the Establishment of a Co-Education Relationship between Parents and Teachers
}

\begin{abstract}
Summary
Co-educational systems contributing to reinforced cooperation between parents and school professionals imply a full agreement between the protagonists, acknowledging equality and mutual consideration of the skills of each partner. This article aims to shed light on the difficulties encountered during the stage of establishment of a co-educational relationship in France. The program under consideration gathers parents of popular background and professional actors of a middle school under the mediation of a community worker. Our analysis discusses the gaps and discrepancies between the will to collaborate and the capacity to overcome one's prejudice concerning a partner, between one's benevolent attention and the actual consideration paid to his/her value, between personal disclosure and dedication to one's role as co-educator.
\end{abstract}

Keywords: Co-education, relationship, teachers, parents, obstacles 